\title{
AVALIAÇÃO DO DESEMPENHO DE VEDAÇÕES VERTICAIS UTILIZANDO O PROCESSO DE ANÁLISE HIERÁRQUICA NA TOMADA DE DECISÃO
}

\section{PERFORMANCE ASSESSMENT OF WALLS USING THE ANALYTIC HIERARCHY PROCESS IN DECISION-MAKING}

\author{
Lucas Rosse Caldas ${ }^{i}$ \\ Universidade Federal do Rio de Janeiro, Rio de Janeiro, RJ, Brasil, lrc.ambiental@ gmail.com \\ Michele Tereza Marques Carvalho ii \\ Universidade de Brasília, Brasília, DF, Brasil, micheletereza@gmail.com
}

\begin{abstract}
Resumo
A avaliação de desempenho de sistemas construtivos tem sido um tema amplamente discutido no setor da construção civil brasileira. Este trabalho teve como objetivo avaliar o desempenho acústico, térmico e ambiental de diferentes sistemas construtivos comumente utilizados em vedações verticais de edificações habitacionais. Foram avaliados os seguintes sistemas: alvenaria de vedação de blocos cerâmicos, alvenaria de tijolos cerâmicos, alvenaria estrutural de blocos de concreto, parede de concreto moldado no local e steel framing. Para o desempenho acústico foram estimados valores do índice de redução sonora ponderado ( $\mathrm{Rw}$ ), enquanto que para o desempenho térmico foram calculados a resistência térmica (Rt) e a capacidade térmica $(\mathrm{Ct})$ dos sistemas construtivos escolhidos. Para a avaliação do desempenho ambiental foram quantificados um indicador de consumo de energia de combustíveis fósseis (IE) e um indicador de emissões de carbono (IC), com base na metodologia de Avaliação do Ciclo de Vida (ACV). Como forma de facilitar a tomada de decisão do projetista foi empregado o Processo de Análise Hierárquica (AHP) para agregar os diferentes critérios avaliados a um indicador de desempenho global (IDG), considerando diferentes cenários. Os resultados mostraram que quando o IDG é calculado o sistema de alvenaria blocos de concreto se apresentou o mais vantajoso para a maioria dos cenários avaliados. Foi possível verificar o potencial do uso do método de AHP para orientar e sistematizar escolhas de diferentes sistemas construtivos para vedações verticais.
\end{abstract}

Palavras-chave: Desempenho de Edificações. Avaliação do Ciclo de Vida. ACV. Processo de Análise Hierárquica.

\begin{abstract}
The performance assessment of construction systems has been a subject widely discussed in the Brazilian building sector. This paper aimed to evaluate the acoustic, thermal and environmental performance of different constructive systems typically used in façades of residential buildings. We assessed the following construction systems: ceramic block masonry, ceramic brick masonry, concrete block structural masonry, reinforced concrete wall and steel framing. For the acoustic performance, the weighted noise reduction index (Rw) was calculated, while for the thermal performance of the thermal resistance (Rt) and the thermal capacity $(\mathrm{Ct})$ were calculated. For the environmental performance assessment, two indicators were quantified: a fossil fuels energy consumption index (IE) and a carbon index (IC), based on the Life Cycle Assessment (LCA) methodology. As a way to facilitate the decision-making process of designers, we used the Analytic Hierarchy Process (AHP) to aggregate the different criteria to a global performance index (GDI). When the GDI is calculated, the concrete block masonry system presented the best results for most of the evaluated scenarios. We verified the potential of using the AHP method to guide and systematize the choice of different constructive systems for walls.
\end{abstract}

Keywords:Building performance. Life Cycle Assessment. LCA. Analytic Hierarchy Process. AHP.

\section{How to cite this article:}

CALDAS, Lucas Rosse; CARVALHO, Michele Tereza Marques. Avaliação do desempenho de vedações verticais utilizando o Processo de Análise Hierárquica na tomada de decisão. PARC Pesquisa em Arquitetura e Construção, Campinas, SP, v. 9, n. 2, P. 109-121, jun. 2018. ISSN 1980-6809. Disponível em: <https://periodicos.sbu.unicamp.br/ojs/index.php/parc/article/view/8651552>. Acesso em: 24 maio 2018. doi:https://doi.org/10.20396/parc.v9i2.8651552. 


\section{Introdução}

A avaliação de desempenho de sistemas construtivos é uma etapa fundamental durante o processo de projeto de edificações (FIGUEIREDO; SILVA, 2010). Desde a publicação da versão mais recente da NBR 15575 (ABNT, 2013), diversos setores da cadeia produtiva da construção civil têm despertado o interesse para esta temática. Essa norma traz requisitos e critérios para a avaliação de desempenho de edificações habitacionais, em diferentes aspectos, entre eles segurança, habitabilidade e sustentabilidade. Pesquisas com foco em desempenho já foram e continuam sendo um tema bastante explorado no Brasil, principalmente voltado para o contexto de habitações de interesse social, que normalmente são os projetos onde a qualidade e o desempenho ficam mais a desejar.

A envoltória, composta por fachadas, piso e cobertura, pode ser considerada um dos principais elementos de uma edificação, sendo responsável pela proteção do ambiente interior contra agentes ambientais externos, como poluição, ruídos, vento, chuva e radiação solar, com papel fundamental nas questões de conforto ambiental e consumo de energia (IWARO; MWASHA, 2013). Neste sentido, a envoltória deve receber atenção especial do projetista durante a fase de projeto, que deve contemplar itens de desempenho, entre eles acústico, térmico, lumínico, estanqueidade, durabilidade, ambiental, entre outros.

Quando se pensa em um projeto que priorize a sustentabilidade ambiental, espera-se que nos sistemas construtivos escolhidos sejam utilizados materiais e componentes que possuam um mínimo impacto ambiental, durante seu ciclo de vida, mas que ao mesmo tempo consigam cumprir requisitos mínimos de desempenho para o uso em foram pensados. Portanto, durante a etapa de especificação de materiais, é interessante que existam indicadores ambientais, calculados com base em Avaliação do Ciclo de Vida (ACV), e que estejam de alguma forma relacionados com critérios de desempenho (CARVALHO; SPOSTO, 2012; CALDAS et al., 2017a).

Tendo em vista a grande quantidade de critérios de desempenho a serem atendidos durante a seleção de um sistema construtivo, o projetista pode se ver perdido neste processo, pois dificilmente um único sistema construtivo conseguirá os melhores resultados para todos os critérios de desempenho. Neste sentido, é necessário que haja alguma forma ou algum tipo de indicador, que seja capaz de mensurar diferentes critérios de uma forma objetiva, para facilitar a tomada de decisão do projetista.

Em função do que foi exposto, neste artigo serão explorados três itens de desempenho presentes na NBR 15575 (ABNT, 2013), sendo eles o desempenho acústico, térmico e ambiental. Através da escolha de diferentes sistemas construtivos para fachadas utilizados no Brasil, buscou-se verificar se os critérios da referida norma são atendidos e ao mesmo tempo verificar as relações entre esses três itens a partir de um indicador de desempenho global (IDG). O IDG proposto tem a função de facilitar a tomada de decisão do projetista, no que concerne a seleção de um sistema construtivo que tenha um bom desempenho global para fachada.

Foram avaliados os seguintes sistemas construtivos: alvenaria de vedação de blocos cerâmicos, alvenaria de tijolos cerâmicos, alvenaria estrutural de blocos de concreto, parede de concreto moldada no local e steel framing.

\section{Fundamentação}

\section{Avaliação de desempenho ambiental, térmico e acústico das edificações}

A NBR 15575 (ABNT, 2013) sugere que sejam avaliados os impactos ambientais ao longo do ciclo de vida dos materiais e sistemas construtivos utilizados nas edificações. Neste sentido, a metodologia de ACV tornase uma proposta bastante interessante pois consegue avaliar diferentes impactos ambientais, ao longo da produção, transporte, uso, manutenção e destinação finalde produtos da construção civil. Tem sido considerada internacionalmente uma das técnicas mais aceitas e completas de avaliação (ANAND; AMOR, 2017; CALDAS et al., 2017a).

A ACV é dividida em quatro etapas, de acordo com a NBR 14040 (ABNT ISO, 2009): (1) definição do objetivo e escopo; (2) análise de inventário; (3) avaliação de impacto do ciclo de vida; (4) interpretação.

Diversos estudos já calcularam as emissões de $\mathrm{CO}_{2}$ e/ou o consumo de energia das edificações, comparando diferentes sistemas construtivos. Taborianski e Prado (2012) compararam as emissões de $\mathrm{CO}_{2}$ de diferentes sistemas construtivos para fachadas, para um escritório em São Paulo. Souza et al. (2016) verificaram os impactos ambientais entre sistemas de alvenaria de blocos cerâmicos, de concreto e paredes de concreto. Caldas et al. (2017b) compararam habitações de alvenaria cerâmica com uma de steel framing. Caldas et al. (2017a) compararam alvenaria cerâmica estrutural com painéis de concreto. Em ambos os estudos se verificou como diferentes desempenhos térmicos impactam nos impactos ambientais ao longo do ciclo de vida das habitações. O sistema de alvenaria de blocos cerâmicos foi vantajoso em ambos os casos.

Do ponto de vista do desempenho térmico Sacht e Rossignolo (2009) compararam o desempenho térmico de sistemas de alvenaria cerâmica e de concreto para 
habitações de interesse social, sendo que o sistema de blocos cerâmicos apresentou melhores resultados. Souza, Amparo e Gomes (2011) avaliaram a importância da capacidade térmica para habitações de steel framing, verificando que a baixa capacidade térmica desse sistema construtivo pode ser indesejada para climas com elevada amplitude térmica. Sorgato et al. (2014) analisaram o método de simulação de desempenho térmico proposto pela NBR 15575 (ABNT, 2013) e verificaram que as condições do piso da edificação em contato com o solo impactam significativamente no desempenho térmico da edificação.

Em todos os estudos os valores de transmitância e capacidade térmica foram calculados, já que servem de dados de entrada para avaliações via simulação, e por isto servem como importantes critérios de desempenho térmico de projeto. A comparação entre diferentes sistemas construtivos também é bastante recorrente nos estudos sobre desempenho térmico de edificações.

Em relação aos estudos sobre desempenho acústico, ainda é um tema pouco explorado nas pesquisas brasileiras, quando comparado com pesquisas referentes ao desempenho térmico. Penedo e Oiticica (2014) avaliaram o nível de isolamento sonoro de duas vedações verticais da de um apartamento em Maceió - AL e concluíram que os níveis de isolamento sonoro aéreo encontrados são inferiores aos recomendados pela norma. Silva Júnior, Rêgo Silva e Pinheiro (2014) avaliaram o desempenho acústico de uma vedação de blocos de gesso, verificando diferenças nos ensaios de campo e laboratório. Santos Filho, Sposto e Caldas (2017) compararam o desempenho acústico entre duas fachadas, sendo uma cortina e outra ventilada, para a cidade de Brasília - DF, observando resultados semelhantes entre as duas fachadas. Percebe-se que os estudos sobre desempenho acústico no ambiente construído, tendem a se concentrar na avaliação de vedações verticais.

Estudos que fazem a ligação entre a avaliação de impactos ambientais ligados ao desempenho acústico de sistemas construtivos ainda são raros na literatura. Tal motivo se dá ao fato desse desempenho não influenciar diretamente o consumo de energia de uma edificação, como ocorre com o desempenho térmico.

\section{Processo de Análise Hierárquica (AHP) para decisão multicritério}

Dentre os diversos métodos existentes para a decisão multicritério, o processo de análise hierárquica, ou do inglês Analytic Hierarchy Process (AHP), desenvolvido por Saaty (1980), é um dos mais utilizados. Esse métodopode ser considerado uma ferramenta de apoio à decisão para problemas multicritério, cujo objetivo é selecionar diferentes alternativas em um processo que considera diversas variáveis (ou critérios), auxiliando assim na tomada de decisão. Nas últimas duas décadas, diversos estudos no setor da construção civil têm utilizado o método de AHP para facilitar a tomada de decisão (SAMPAIO; LIMA; BARROS NETO, 2010; DARKO et al., 2018)

Sampaio, Lima e Barros Neto (2010) avaliaram diferentes métodos de tomada de decisão para auxiliar o desenvolvimento de projetos arquitetônicos, elencando os aspectos positivos e negativos de cada método. Eles verificaram que necessidades insatisfatórias dos usuários podem ser priorizadas com o auxílio do AHP.

Marchezetti, Kaviski e Braga (2011) aplicaram o método para a avaliação de diferentes alternativas tecnológicas para o tratamento de resíduos sólidos domésticos. Carvalho e Sposto (2012) desenvolveram a metodologia de Avaliação de Sustentabilidade para habitações de interesse social (MASP-HIS), também propondo indicadores de projeto para diferentes combinações de vedações verticais, mas com foco nas questões ambientais, econômicas e sociais.

Mattana et al. (2012) também aplicaram o método para a decisão entre diferentes tipos de agregados miúdos para confecção de argamassa, apresentando no final um indicador de desempenho global. Santos e Cruz (2013) compararam diferentes sistemas construtivos para lajes de edifícios comerciais, sendo que a laje steel deck foi a mais vantajosa.

Grünberg, Medeiros e Tavares (2014) utilizaram o AHP para comparar diferentes selos de certificações ambientais para edificações, sendo elas o Leadership in Energy and Environmental Design (LEED), Alta Qualidade Ambiental (AQUA) e Selo Casa Azul. O último selo se apresentou o mais vantajoso para realidade brasileira. Franzen (2015) utilizou a AHP para subsidiar a escolha de diferentes configurações de steel framing, utilizando critérios de desempenho térmico, acústico e custos.

Darko et al. (2018) realizaram um estado da arte de estudos que utilizaram o AHP para apoiar a decisão de problemas relacionados à construção civil e verificaram duas principais tendências: gerenciamento de riscos e construção sustentável.

A partir desses estudos verifica-se que o método de AHP pode ser bastante eficaz para apoiar a tomada de decisão de diversos tipos de problemas relacionados ao setor da construção civil.

\section{Método}

O método de pesquisa utilizado no presente estudo se enquadra como estudo de caso, realizado com base em pesquisa bibliográfica e documental. 


\section{Descrição dos sistemas construtivos avaliados}

No Quadro 1 são apresentados os cinco sistemas construtivos avaliados neste estudo: alvenaria de blocos cerâmicos, de tijolos cerâmicos e blocos de concreto estrutural, parede de concreto moldada no local e steel framing.

\begin{tabular}{|c|c|c|}
\hline Sistema construtivo & |lustração & $\begin{array}{l}\text { Massa } \\
\left(\mathrm{kg} / \mathrm{m}^{2}\right)\end{array}$ \\
\hline $\begin{array}{l}\text { Alvenaria de blocos cerâmicos } \\
9 \times 19 \times 19 \mathrm{~cm} \text { com revestimento de } \\
2,5 \mathrm{~cm}-19,0 \mathrm{~cm}\end{array}$ & & 185,5 \\
\hline $\begin{array}{l}\text { Alvenaria de tijolos cerâmicos } \\
10 \times 6 \times 22 \mathrm{~cm}-10,0 \mathrm{~cm}\end{array}$ & & 164,3 \\
\hline $\begin{array}{l}\text { Alvenaria de blocos de concreto } \\
\text { 14x19x39 cm com revestimento de } \\
2,5 \mathrm{~cm}-19,0 \mathrm{~cm}\end{array}$ & & 266,4 \\
\hline $\begin{array}{l}\text { Parede de concreto armado } \\
\text { moldada no local com } 12,0 \mathrm{~cm}\end{array}$ & & 309,5 \\
\hline $\begin{array}{c}\text { Steel framing, com revestimento em } \\
\text { placas cimentícias (externamente de } \\
1,00 \mathrm{~cm} \text { ), drywall (internamente de } \\
1,25 \mathrm{~cm} \text { ), chapas de OSB (uma de } \\
1,11 \mathrm{~cm} \text { e outra de } 0,95 \mathrm{~cm} \text { ) e lã de } \\
\text { rocha }(7,0 \mathrm{~cm})-13,3 \mathrm{~cm}\end{array}$ & & 75,4 \\
\hline
\end{tabular}

Fonte: Os autores. ${ }^{1}$ Retirado do MMA (2018).

\section{Avaliação do Desempenho Acústico}

A isolação acústica de paredes maciças foi estimada pela Equação (1) (CBIC, 2013):

$$
R w=12+M^{1 / 3}
$$

Onde, Rw - Índice de redução sonora ponderado $(\mathrm{dB})$ e $\mathrm{M}$ - Massa da vedação vertical $\left(\mathrm{kg} / \mathrm{m}^{2}\right)$.

Para o sistema de steel framing foi utilizado o valor apresentado por Radavelli e Paul (2015), que calcularam o $\mathrm{Rw}$ para um sistema de steel framing com as mesmas camadas consideradas neste estudo.
De posse dos valores de $\mathrm{Rw}$ dos sistemas de vedação vertical escolhidos, foi verificado o atendimento a NBR 15575 (ABNT, 2013), de acordo com o critério - "Níveis de desempenho de componentes para fachada, para ensaios em laboratório".

O mesmo método, tanto para estimativa de Rw como para verificação do atendimento à norma, foi utilizado no estudo publicado pelo Sinduscon (2013).

\section{Avaliação do Desempenho Térmico}

Neste estudo foi empregado somente o procedimento 1 da NBR 15575 (ABNT, 2013), denominado de simplificado ou normativo. O requisito avaliado é denominado de "Adequação as paredes externas". Os valores de U e Ct foram calculados de acordo com o procedimento de cálculo descrito na NBR 15220-2 (ABNT, 2005).

\section{Avaliação do Desempenho Ambiental}

Para a avaliação do desempenho ambiental foi utilizada a metodologia de ACV, com suas etapas detalhadas a seguir.

(1) Definição do objetivo e escopo

Este estudo tem como objetivo a quantificação de indicadores de desempenho ambiental para diferentes sistemas construtivos utilizados em fachadas, para a realidade brasileira.

Foram escolhidos dois indicadores de desempenho ambiental, sendo eles o indicador de consumo de energia de combustíveis fósseis (IE) e o indicador de emissões de carbono (IC), que são os mais utilizados nos estudos de ACVaplicados a edificações (CABEZA et al., 2014; CHAU; LEUNG; NG, 2015).

O estudo seguiu as diretrizes da NBR 14040 (ABNT ISO, 2009). A norma EN 15978 (ECS, 2011) foi desenvolvida na Comunidade Europeia com o objetivo de padronizar a aplicação da ACV no setor da construção civil (ANAND; AMOR, 2017). Dessa forma, no presente estudo ela foi adotada, sendo que o escopo foi definido como do tipo "berço ao portão" considerando os estágios de produção dos materiais utilizados nos sistemas construtivos.

A unidade funcional escolhida foi a área de vedação vertical $\left(\mathrm{em} \mathrm{m}^{2}\right)$, que é comumente escolhida quando se avalia diferentes sistemas construtivos (CABEZA et al., 2014).

\section{(2) Análise do inventário}

Para a quantificação dos materiais por $\mathrm{m}^{2}$ de fachada, foram utilizados dados do Sistema Nacional de Pesquisa de Custos e Índices da Construção Civil (SINAPI) e da Tabela de Composição de Preços para Orçamentos (TCPO, 2012). Ao final, todos os materiais foram convertidos em massa $\left(\mathrm{kg} / \mathrm{m}^{2}\right)$, a partir de dados de fabricantes. 
Como o Brasil possui um banco de dados ainda pouco desenvolvido para o setor da construção civil, foi utilizado o banco de dados internacional Ecoinvent v. 3.3, com algumas adaptações para a realidade brasileira. Como, por exemplo, para cimento e concreto foi possível utilizar a Declaração Ambiental de Produto (DAP) da Votorantim Cimentos (INTERNATIONAL EPD SYSTEM, 2018). As misturas de argamassas, para chapisco, camada única e assentamento dos blocos foram realizadas de acordo com as práticas utilizadas no Brasil.

(3) Avaliação do impacto do ciclo de vida

Neste estudo foi escolhido o método de avaliação do impacto do ciclo de vida (AICV) CML, linha de base IA, versão 3.03 .

Foram consideradas as seguintes categorias de impacto ambiental: Depleção de Combustíveis Fósseis (ADP-ff) para o indicador IE, em $\mathrm{MJ} / \mathrm{m}^{2}$, e o Potencial de Aquecimento Global (GWP100) para o indicador IC, em $\mathrm{kgCO}_{2 \mathrm{e}}{ }^{1 /} \mathrm{m}^{2}$, de acordo com a EN 15978 (ECS, 2011).

As Equações (2) e (3) mostram o cálculo dos indicadores de desempenho ambiental. Na Tabela 1 são apresentados os dados utilizados para o cálculo dos indicadores ambientais.

$$
I E=\sum_{i=1}^{n} m_{i} x\left(1+\frac{P i}{100}\right) x F A D P-f f_{i}
$$

Onde,IE- indicador de consumo de energia de fontes fósseis $\left(\mathrm{MJ} / \mathrm{m}^{2}\right)$; i - material de construção discriminado; $\mathrm{n}$ - número de materiais; $\mathrm{m}$ - massa do material utilizado na fachada $\left(\mathrm{kg} / \mathrm{m}^{2}\right) ; \mathrm{P}$ - perdas dos materiais (\%) e FADP$\mathrm{ff}$ - fator de depleção de combustíveis fósseis (MJ/kg).

$$
I C=\sum_{i=1}^{n} m_{i} x\left(1+\frac{P i}{100}\right) x F G W P_{i}
$$

Onde,IC - indicador de emissões de carbono $\left(\mathrm{kgCO}_{2 \mathrm{e}} / \mathrm{m}^{2}\right)$; i - material de construção discriminado; $n$ - número de materiais; $\mathrm{m}$ - massa do material utilizado na fachada $\left(\mathrm{kg} / \mathrm{m}^{2}\right) ; \mathrm{P}$ - perdas dos materiais (\%) e FGWP - fator de potencial de aquecimento global $\left(\mathrm{kgCO}_{2 \mathrm{e}} / \mathrm{kg}\right)$.

\section{Método Analytic Hierarchy Process (AHP) para a tomada de decisão}

Utilizou-se o método AHP, desenvolvido por Saaty (1980), como ferramenta de apoio à tomada de decisão multicritério para a seleção do sistema construtivo que apresente o melhor desempenho global, como é apresentado na Figura 1. Ao final, os diferentes critérios de desempenho foram agregados a um único indicador,

\begin{tabular}{|c|c|c|c|c|}
\hline Materiais & $\mathrm{m}\left(\mathrm{kg} / \mathrm{m}^{2}\right)$ & $\begin{array}{c}\text { Perdas } \\
(\%)\end{array}$ & $\begin{array}{l}\text { FADP-ff } \\
(\mathrm{MJ} / \mathrm{kg})\end{array}$ & $\begin{array}{c}\text { FGWP } \\
\left(\mathrm{kgCO}_{2 \mathrm{e}} \mathrm{l}\right. \\
\mathrm{kg})\end{array}$ \\
\hline \multicolumn{5}{|c|}{ Alvenaria de blocos cerâmicos } \\
\hline Bloco cerâmico & 62,5 & $5 \%$ & 2,00 & 0,22 \\
\hline Chapisco & 19 & $10 \%$ & 1,22 & 0,20 \\
\hline Argamassa mista & 104 & $10 \%$ & 1,21 & 0,19 \\
\hline \multicolumn{5}{|c|}{ Tijolos cerâmicos } \\
\hline Tijolos cerâmicos & 132 & 0,03 & 2,00 & 0,22 \\
\hline Argamassa mista & 32 & 0,1 & 1,21 & 0,19 \\
\hline \multicolumn{5}{|c|}{ Alvenaria de blocos de concreto } \\
\hline Bloco de concreto & 156 & $3 \%$ & 0,88 & 0,13 \\
\hline Aço & 0,4 & $5 \%$ & 20,02 & 2,04 \\
\hline Chapisco & 19 & $10 \%$ & 1,22 & 0,20 \\
\hline Argamassa mista & 91 & $10 \%$ & 1,21 & 0,19 \\
\hline \multicolumn{5}{|c|}{ Parede de concreto } \\
\hline Concreto & 300 & $5 \%$ & 0,6 & 0,08 \\
\hline Aço & 9,48 & $5 \%$ & 20,01917 & 2,04 \\
\hline Alumínio & 0,063 & $0 \%$ & 171,4192 & 17,39 \\
\hline \multicolumn{5}{|c|}{ Steel framing } \\
\hline Perfil de aço & 27,1 & $3 \%$ & 27,64 & 2,71 \\
\hline Parafusos & 0,3 & $0 \%$ & 27,64 & 2,71 \\
\hline Massa rejunte & 0,4 & $5 \%$ & 1,13 & 0,16 \\
\hline Lã de rocha & 2,2 & $0 \%$ & 15,27 & 1,34 \\
\hline Chapa de OSB & 14,1 & $5 \%$ & 7,42 & 0,43 \\
\hline Placa cimentícia & 18,9 & $0 \%$ & 10,00 & 1,24 \\
\hline Placa de gesso & 12,5 & $5 \%$ & 4,03 & 0,00 \\
\hline
\end{tabular}
chamado de Indicador de Desempenho Global (IDG).
Fonte: Os autores.

Figura 1 - Rede de critérios e alternativas de escolha dométodo AHP deste estudo

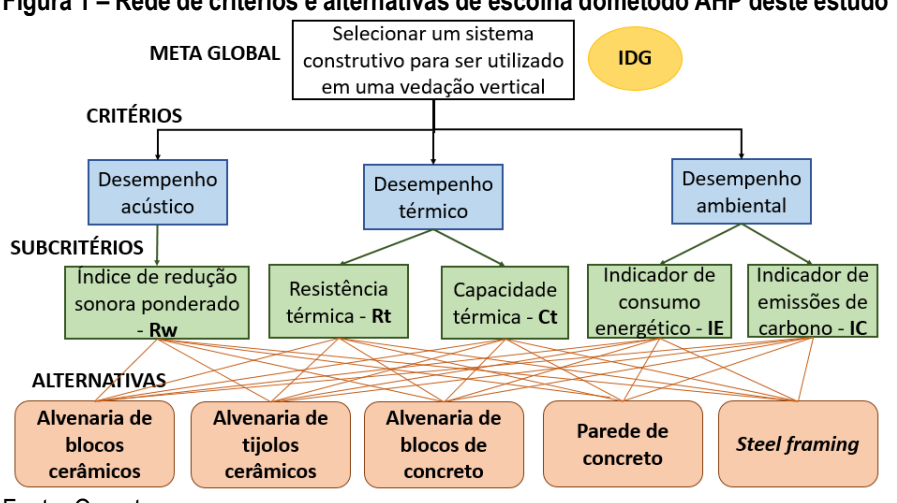

Fonte: Os autores.

A aplicação doAHP foi dividida nas seguintes etapas: (1) definição das alternativas (os sistemas construtivos a serem avaliados); (2) definição dos critérios (acústico, térmicos e ambientais) e subcritérios; (3) avaliação da importância de cada subcritério (definição das matrizes de decisão); (4) cálculo do IDG.

Foram utilizados cinco critérios: (1) Índice de redução sonora ponderado (Rw); (2) Capacidade térmica $(\mathrm{Ct})$; (3) Resistência térmica (Rt); (4) Inverso do indicador de emissões de carbono (1/IC); (5) Inverso do indicador de consumo de energia (1/IE). Sendo que os critérios número (2) e (3) já foram utilizados no estudo de Frazen (2015). 
Foi calculado o inverso dos valores dos indicadores ambientais com o intuito de que os critérios sigam a mesma tendência de serem diretamente proporcionais ao desempenho, mesmo procedimento utilizado por Mattana et al. (2012).

Para a realização do julgamento dos critérios, foram adotados os valores recomendados pela ASTM E 1765 (ASTM, 2002), apresentados na Tabela 2, também utilizado por Mattana et al. (2012) e Cruz e Souza (2013). Essa norma traz as diretrizes e recomendações para o uso da AHP quando aplicado a edificações e suas partes.

É comum no uso do AHP a entrevista com especialistas para a determinação de quão importante é um critério específico em relação ao outro para se atingir a meta desejada (CRUZ; SOUZA, 2013). No entanto, para o presente estudo optou-se por tratar de cenários a fim de verificar diferentes opções de projeto e apresentar a forma de aplicação e a versatilidade do método de AHP para sistematizar a tomada de decisão para a seleção de sistemas construtivos com diferentes critérios de desempenho. No estudo de Marchezetti, Kaviski e Braga (2011) os próprios autores definiram os pesos dos critérios, sendo também o método utilizado no presente estudo.

Tabela 2 - Escalas de importância para os critérios analisados

\begin{tabular}{cc}
\hline Escalas de comparação & Valor \\
\hline A igual importância que B & 1 \\
\hline A pouco mais importante que B & 3 \\
\hline A mais importante que B & 5 \\
\hline A muito mais importante que B & 7 \\
\hline A extremamente mais importante que B & 9 \\
\hline
\end{tabular}

Fonte: ASTM E 1765 (ASTM, 2002).

Foram avaliados seis cenários:

- Cenário 1 - Avaliação multicritério sem dar peso para os critérios escolhidos: relação de igual importância para todos - peso igual a 1 .

- Cenário 2 - Com maior peso para o critério acústico. Relação acústico/térmico - peso 5; Relação acústico/ambiental - peso 5; Relação térmico/ambiental - peso 1 .

- Cenário 3 - com maior peso para os critérios térmicos $(\mathrm{Ct}=\mathrm{Rt})$. Relação térmico/acústico - peso 5; Relação térmico/ambiental - peso 5; Relação acústico/ambiental - peso 1 .

- Cenário 4 - com maior peso para os critérios térmicos (sendo $\mathrm{Ct}>\mathrm{Rt}$ ). Relação $\mathrm{Ct} / \mathrm{Rt}$ - peso 3; Relação $\mathrm{Ct} / \mathrm{Rw}$ - peso 5; Relação Rt/Rw - peso 3; Relação Ct/Ambiental - peso 5; Relação Rt/ Ambiental - peso 3; Relação acústico/ambiental - peso 1 .

- Cenário 5 - com maior peso para os critérios térmicos (sendo $\mathrm{Ct}<\mathrm{Rt}$ ). Relação Ct/Rt - peso $1 / 3$; Relação
$\mathrm{Ct} / \mathrm{Rw}$ - peso 3; Relação Rt/Rw - peso 5; Relação Ct/Ambiental - peso 3; Relação Rt/ Ambiental - peso 5; Relação acústico/ambiental - peso 1.

- Cenário 6 - com maior peso para os critérios ambientais. Relação ambiental/acústico - peso 5; Relação ambiental/térmico - peso 5; Relação acústico/térmico - peso 1 .

As matrizes de decisão com os vetores Eigen para cada cenário estão apresentadas nas Tabelas $3,4,5,6,7$ e 8 respectivamente.

$\mathrm{O}$ vetor de Eigen apresenta os pesos relativos entre os critérios avaliados. Ele é obtido por meio da média aritmética dos valores de cada um dos critérios, totalizando sempre 100\% (SANTOS; CRUZ, 2013).

\begin{tabular}{ccccccc}
\hline \begin{tabular}{l} 
Tabela 3-Matriz de decisão-cenário 1 \\
\hline Critérios
\end{tabular} & $\mathrm{Rw}$ & $\mathrm{Ct}$ & $\mathrm{Rt}$ & $1 / \mathrm{IC}$ & $1 / \mathrm{IE}$ & $\begin{array}{c}\text { Vetor } \\
\text { Eigen }\end{array}$ \\
\hline $\mathrm{Rw}$ & 1 & 1 & 1 & 1 & 1 & $20,0 \%$ \\
\hline $\mathrm{Ct}$ & 1 & 1 & 1 & 1 & 1 & $20,0 \%$ \\
\hline $\mathrm{Rt}$ & 1 & 1 & 1 & 1 & 1 & $20,0 \%$ \\
\hline $1 / \mathrm{IC}$ & 1 & 1 & 1 & 1 & 1 & $20,0 \%$ \\
\hline $1 / \mathrm{IE}$ & 1 & 1 & 1 & 1 & 1 & $20,0 \%$ \\
\hline Total & 5,0 & 5,0 & 5,0 & 5,0 & 5,0 & $100 \%$ \\
\hline
\end{tabular}

Nota: $\mathrm{Cl}=0,0 \mathrm{CR}=0,0$.Fonte: Os autores.

\begin{tabular}{ccccccc}
\hline \multicolumn{7}{l}{ Tabela 4-Matriz de decisão-cenário 2 } \\
\hline Critérios & $\mathrm{Rw}$ & $\mathrm{Ct}$ & $\mathrm{Rt}$ & $1 / \mathrm{IC}$ & $1 / \mathrm{IE}$ & $\begin{array}{c}\text { Vetor } \\
\text { Eigen }\end{array}$ \\
\hline $\mathrm{Rw}$ & 1 & 5 & 5 & 5 & 5 & $55,6 \%$ \\
\hline $\mathrm{Ct}$ & $1 / 5$ & 1 & 1 & 1 & 1 & $11,1 \%$ \\
\hline $\mathrm{Rt}$ & $1 / 5$ & 1 & 1 & 1 & 1 & $11,1 \%$ \\
\hline $1 / \mathrm{IC}$ & $1 / 5$ & 1 & 1 & 1 & 1 & $11,1 \%$ \\
\hline $1 / \mathrm{IE}$ & $1 / 5$ & 1 & 1 & 1 & 1 & $11,1 \%$ \\
\hline Total & 1,8 & 9,0 & 9,0 & 9,0 & 9,0 & $100 \%$ \\
\hline
\end{tabular}

Nota: $\mathrm{Cl}=0,0 \mathrm{CR}=0,0$.Fonte: Os autores.

\begin{tabular}{ccccccc}
\multicolumn{2}{l}{ Tabela5 - Matriz de decisão-cenário 3 } \\
\hline Critérios & $\mathrm{Rw}$ & $\mathrm{Ct}$ & $\mathrm{Rt}$ & $1 / \mathrm{IC}$ & $1 / \mathrm{IE}$ & $\begin{array}{c}\text { Vetor } \\
\text { Eigen }\end{array}$ \\
\hline $\mathrm{Rw}$ & 1 & $1 / 5$ & $1 / 5$ & 1 & 1 & $7,7 \%$ \\
\hline $\mathrm{Ct}$ & 5 & 1 & 1 & 5 & 5 & $38,5 \%$ \\
\hline $\mathrm{Rt}$ & 5 & 1 & 1 & 5 & 5 & $38,5 \%$ \\
\hline $1 / \mathrm{IC}$ & 1 & $1 / 5$ & $1 / 5$ & 1 & 1 & $7,7 \%$ \\
\hline $1 / \mathrm{IE}$ & 1 & $1 / 5$ & $1 / 5$ & 1 & 1 & $7,7 \%$ \\
\hline Total & 13,0 & 2,6 & 2,6 & 13,0 & 13,0 & $100 \%$ \\
\hline
\end{tabular}

Nota: $\mathrm{Cl}=0,0 \mathrm{CR}=0,0$. Fonte: Os autores.

Tabela 6 - Matriz de decisão - cenário 4
\begin{tabular}{ccccccc}
\hline Critérios & $\mathrm{RW}$ & $\mathrm{Ct}$ & $\mathrm{Rt}$ & $1 / \mathrm{IC}$ & $1 / \mathrm{IE}$ & $\begin{array}{c}\text { Vetor } \\
\text { Eigen }\end{array}$ \\
\hline $\mathrm{Rw}$ & 1 & $1 / 5$ & $1 / 3$ & 1 & 1 & $8,9 \%$ \\
\hline $\mathrm{Ct}$ & 5 & 1 & 3 & 5 & 5 & $49,6 \%$ \\
\hline $\mathrm{Rt}$ & 3 & $1 / 3$ & 1 & 3 & 3 & $23,8 \%$ \\
\hline $1 / \mathrm{IC}$ & 1 & $1 / 5$ & $1 / 3$ & 1 & 1 & $8,9 \%$ \\
\hline $1 / \mathrm{IE}$ & 1 & $1 / 5$ & $1 / 3$ & 1 & 1 & $8,9 \%$ \\
\hline Total & 11,0 & 1,9 & 5,0 & 11,0 & 11,0 & $100 \%$ \\
\hline
\end{tabular}

Nota: $\mathrm{Cl}=0,011 \mathrm{CR}=0,09$.Fonte: Os autores 
Tabela 7 - Matriz de decisão - cenário 5

\begin{tabular}{ccccccc}
\hline Critérios & $\mathrm{Rw}$ & $\mathrm{Ct}$ & $\mathrm{Rt}$ & $1 / \mathrm{IC}$ & $1 / \mathrm{IE}$ & $\begin{array}{c}\text { Vetor } \\
\text { Eigen }\end{array}$ \\
\hline $\mathrm{Rw}$ & 1 & $1 / 3$ & $1 / 5$ & 1 & 1 & $8,9 \%$ \\
\hline $\mathrm{Ct}$ & 3 & 1 & $1 / 3$ & 3 & 3 & $23,8 \%$ \\
\hline $\mathrm{Rt}$ & 5 & 3 & 1 & 5 & 5 & $49,6 \%$ \\
\hline $1 / \mathrm{IC}$ & 1 & $1 / 3$ & $1 / 5$ & 1 & 1 & $8,9 \%$ \\
\hline $1 / \mathrm{IE}$ & 1 & $1 / 3$ & $1 / 5$ & 1 & 1 & $8,9 \%$ \\
\hline Total & 11,0 & 5,0 & 1,9 & 11,0 & 11,0 & $100 \%$ \\
\hline
\end{tabular}

Nota: $\mathrm{Cl}=0,011 \mathrm{CR}=0,09$.Fonte: Os autores.

Tabela 8 - Matriz de decisão - cenário 6

\begin{tabular}{ccccccc}
\hline Critérios & Rw & Ct & Rt & $1 / \mathrm{IC}$ & $1 / / \mathrm{E}$ & $\begin{array}{c}\text { Vetor } \\
\text { Eigen }\end{array}$ \\
\hline $\mathrm{Rw}$ & 1 & 1 & 1 & $1 / 5$ & $1 / 5$ & $7,7 \%$ \\
\hline $\mathrm{Ct}$ & 1 & 1 & 1 & $1 / 5$ & $1 / 5$ & $7,7 \%$ \\
\hline $\mathrm{Rt}$ & 1 & 1 & 1 & $1 / 5$ & $1 / 5$ & $7,7 \%$ \\
\hline $1 / \mathrm{IC}$ & 5 & 5 & 5 & 1 & 1 & $38,5 \%$ \\
\hline $1 / \mathrm{IE}$ & 5 & 5 & 5 & 1 & 1 & $38,5 \%$ \\
\hline Total & 13,0 & 13,0 & 13,0 & 2,6 & 2,6 & $100 \%$ \\
\hline
\end{tabular}

Nota: $\mathrm{Cl}=0,0 \mathrm{CR}=0,0$. Fonte: Os autores.

Como uma avaliação adicional, pensando em uma forma de penalizar os sistemas construtivos que não atendem aos critérios mínimos da NBR 15575 (ABNT, 2013), foi calculado o IDG considerando como zero a contribuição de dado sistema construtivo para o critério não atendido. Por exemplo, se o sistema de steel framing não atende à norma no critério de capacidade térmica $(\mathrm{Ct})$, a contribuição desse critério é zerada no cálculo do IDG.

Para cada cenário foram calculadas as taxas de consistência (CR), a partir do cálculo dos índices de consistência (IC) que se relaciona com os índices randômicos (IR).De acordo com Saaty (1991), para uma amostra de cinco elementos utiliza-se o valor de 1,12 . As equações utilizadas para o cálculo de IC e CR podem ser verificadas em Saaty (1991), Marchezetti, Kaviski e Braga (2011)e Souza e Cruz (2013).

A partir dos resultados dos critérios utilizados ( $\mathrm{Rw}, \mathrm{Ct}$, Rt, 1/IC e 1/IE)foi calculada uma matriz com valores variando de 0 a 1, adotando como 1 o maior valor de cada critério, sendo os demais calculados a partir da divisão pelo maior valor. Os valores da matriz foram multiplicados pelos valores do vetor Eigen de cada cenário como uma média ponderada, apresentada na Equação (4).

$$
I D G=\frac{\sum_{i=1}^{n} c_{i} x V E i}{\sum_{i=1}^{n} V E i}
$$

Onde,IDG - indicador de desempenho global;i - critério discriminado; $\mathrm{n}$ - número de critérios; $\mathrm{c}$ - critério utilizado, com valores variando de 0 a 1 (Rw, Ct, Rt, 1/IC, 1/IE) e $\mathrm{VE}$ - Vetor Eigen para cada cenário avaliado.

\section{Resultados e Discussão}

\section{Avaliação do Desempenho Acústico}

Na Tabela 9 são apresentados os valores de Rw e a classificação nos níveis de atendimento da NBR 15575 (ABNT, 2013).

\begin{tabular}{ccccc}
\multicolumn{6}{c}{ Tabela 9 - Verificação do atendimento dos critérios de desempenho acústico } \\
\hline Sistemas & Rw $(\mathrm{dB})$ & Classe I & Classe II & $\begin{array}{c}\text { Classe } \\
\text { III }\end{array}$ \\
\hline $\begin{array}{c}\text { Alvenaria de blocos } \\
\text { cerâmicos }\end{array}$ & 42,2 & $\mathrm{~S}$ & $\mathrm{~S}$ & $\mathrm{I}$ \\
\hline Tijolos cerâmicos & 41,0 & $\mathrm{~S}$ & $\mathrm{~S}$ & $\mathrm{I}$ \\
\hline $\begin{array}{c}\text { Alvenaria de blocos de } \\
\text { concreto }\end{array}$ & 46,1 & $\mathrm{~S}$ & $\mathrm{~S}$ & $\mathrm{~S}$ \\
\hline Parede de concreto & 47,9 & $\mathrm{~S}$ & $\mathrm{~S}$ & $\mathrm{~S}$ \\
\hline Steel framing & 47,0 & $\mathrm{~S}$ & $\mathrm{~S}$ & $\mathrm{~S}$ \\
\hline
\end{tabular}

Fonte: Os autores. S - Nivel Superior; I - Nível Intermediário.

Observa-se que, entre os materiais cerâmicos e de concreto, os últimos apresentaram maior $\mathrm{Rw}$, o que era de se esperar, tendo em vista que são materiais de maior massa específica e, consequentemente, pela Lei das Massas, quanto maior a massa, maior é o potencial de isolamento acústico. Já o steel framing funciona por um princípio diferente, devido ao processo de impedância acústica.

Santos Filho, Sposto e Caldas (2017) avaliaram uma fachada cortina e outra ventilada e verificaram desempenhos semelhantes, sendo que os mecanismos de isolamento acústico são diferentes, a primeira também pela massa e a segunda por diferenças de impedâncias das camadas da fachada.

\section{Avaliação do Desempenho Térmico}

$\mathrm{Na}$ Tabela 10 os valores de $\mathrm{U}$ e $\mathrm{Ct}$ dos sistemas construtivos são apresentados.

Tabela 10 - Verificação do atendimento dos critérios de desempenho térmico

\begin{tabular}{|c|c|c|c|}
\hline Sistemas & $\underset{\left(W / m^{2} . K\right)}{U}$ & $\mathrm{Ct}\left(\mathrm{kJ} / \mathrm{m}^{2} . \mathrm{K}\right)$ & Atendimento à norma \\
\hline $\begin{array}{l}\text { Alvenaria de } \\
\text { blocos } \\
\text { cerâmicos }\end{array}$ & 2,37 & 151,0 & $\begin{array}{l}\text { Atende todas as zonas } \\
\text { bioclimáticas }\end{array}$ \\
\hline $\begin{array}{l}\text { Tijolos } \\
\text { cerâmicos }\end{array}$ & 3,65 & 158,0 & $\begin{array}{c}\text { Atende as zonas } \\
\text { bioclimáticas } 3 \text { a } 8 \text {. } \\
\text { Absortância deverá ser } \\
\text { menor que } 0,6\end{array}$ \\
\hline $\begin{array}{l}\text { Alvenaria de } \\
\text { blocos de } \\
\text { concreto }\end{array}$ & 2,68 & 272,0 & $\begin{array}{c}\text { Atende as zonas } \\
\text { bioclimáticas } 3 \text { a } 8 \text {. } \\
\text { Absortância deverá ser } \\
\text { menor que } 0,6\end{array}$ \\
\hline $\begin{array}{l}\text { Parede de } \\
\text { concreto }\end{array}$ & 4,19 & 288,0 & Não atende \\
\hline Steel framing & 0,66 & 58,6 & $\begin{array}{c}\text { Atende somente a Zona } \\
\text { Bioclimática } 8\end{array}$ \\
\hline
\end{tabular}

Fonte: Os autores. 
Diferentemente do critério de desempenho acústico, por este procedimento não é possível fazer uma classificação em diferentes níveis.

\section{Avaliação do Desempenho Ambiental}

Avaliando os resultados, verifica-se que não são todos os sistemas construtivos que atendem aos critérios de desempenho térmico da NBR 15575 (ABNT, 2013), sendo que alguns atendem somente para algumas zonas bioclimáticas. Por exemplo, o steel framing consegue atender o critério somente para a Zona Bioclimática 8 , pois para atender as outras zonas precisaria ter no mínimo 130 $\mathrm{kJ} / \mathrm{m}^{2} . \mathrm{K}$ de capacidade térmica. A partir desses resultados, o sistema de parede de concreto terá seu valor de Rt zerado e o steel framing terá o valor Rt zerado para o cálculo de seus IDGs.

Nas Figuras 2 e 3 são apresentados os resultados dos indicadores ambientais avaliados.

Figura 2 - Comparação do indicador de consumo de energia (IE) dos sistemas construtivos



Fonte: Os autores.

Figura 3 - Comparação do indicador de emissões de carbono (IC) sistemas construtivos



Fonte: Os autores.

Nota-se que os sistemas com alvenaria em cerâmica foram os que apresentaram menores resultados de consumo de energia e emissões de $\mathrm{CO}_{2}$, o que está alinhado com Souza et al. (2016) e Caldas et al. (2017a). Nestes sistemas, a maior parte dos impactos ambientais se dá pelo maior consumo de argamassa e, consequentemente, cimento, o que levou o sistema de blocos cerâmicos com revestimento argamassado a ter um maior indicador de carbono (IC), se comparado ao sistema de tijolos aparentes. O steel framing apresentou elevados impactos ambientais, principalmente devido à produção de aço galvanizado dos perfis.

Os sistemas que usam concreto também têm seus impactos ambientais influenciados pelo cimento, enquanto que a parede de concreto, especificamente, sofre influência das fôrmas de alumínio.

É importante ressaltar que, para que os resultados sejam mais assertivos, seria interessante fazer uma análise de incertezas dos dados utilizados nos sistemas de concreto e cerâmica.

\section{Avaliação Considerando o Indicador de Desempenho Global (IDG)}

$\mathrm{Na}$ Tabela 11 são apresentados os valores dos critérios utilizados no AHP normalizados pelo maior valor, com intervalo variando de 0 a 1 .

Tabela 11 - Resultados dos critérios avaliados (intervalo de 0 a 1 )

\begin{tabular}{cccccc}
\hline Alternativas & Rw & Ct & Rt & 1/IC & 1/IE \\
\hline Blocos cerâmicos & 0,754 & 0,524 & 0,278 & 1,000 & 0,914 \\
\hline Tijolos cerâmicos & 0,733 & 0,549 & 0,181 & 0,937 & 1,000 \\
\hline Blocos de concreto & 0,823 & 0,944 & 0,246 & 0,993 & 0,826 \\
\hline Parede de concreto & 0,855 & 1,000 & 0,158 & 0,740 & 0,763 \\
\hline Parede de concreto* & 0,855 & 1,000 & 0,000 & 0,740 & 0,763 \\
\hline Steel framing & 1,000 & 0,203 & 1,000 & 0,254 & 0,321 \\
\hline Steel framing & 1,000 & 0,000 & 1,000 & 0,254 & 0,321 \\
\hline
\end{tabular}

Nota: * Sistemas que tiveram algum critério zerado por não atender o critério mínimo da NBR 15575 (ABNT,2013). Fonte: Os autores.

Os valores de IDG, apresentados nas Figuras 3, 4, 5, 6, 7, e 8 para os seis cenários avaliados, foram obtidos a partir da aplicação da Equação (4). A cor verde representa o melhor resultado e a vermelha o pior.

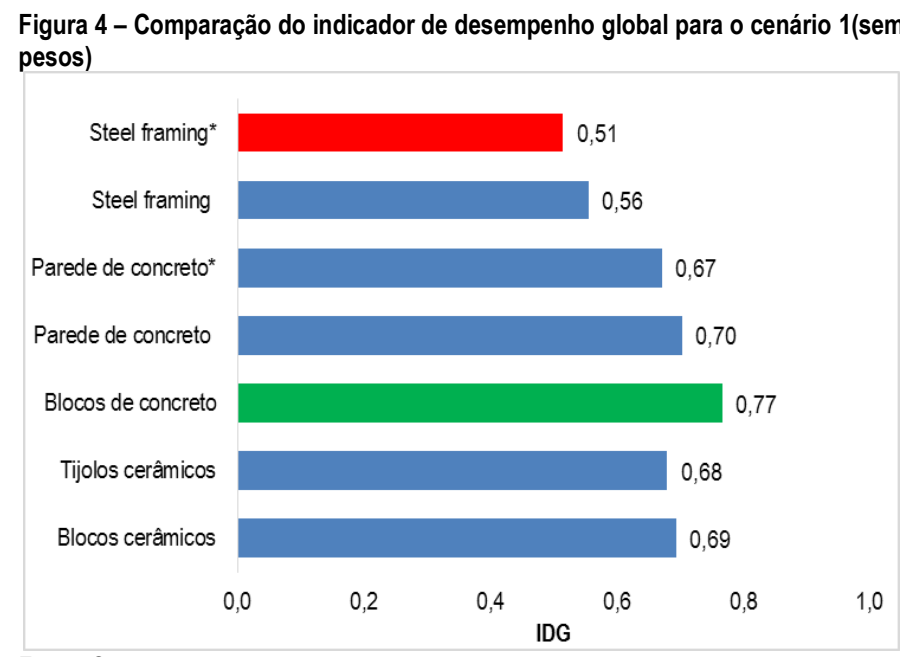

Fonte: Os autores. 
Figura 5 - Comparação do indicador de desempenho global para o cenário 2 (com maior peso para o critério acústico)

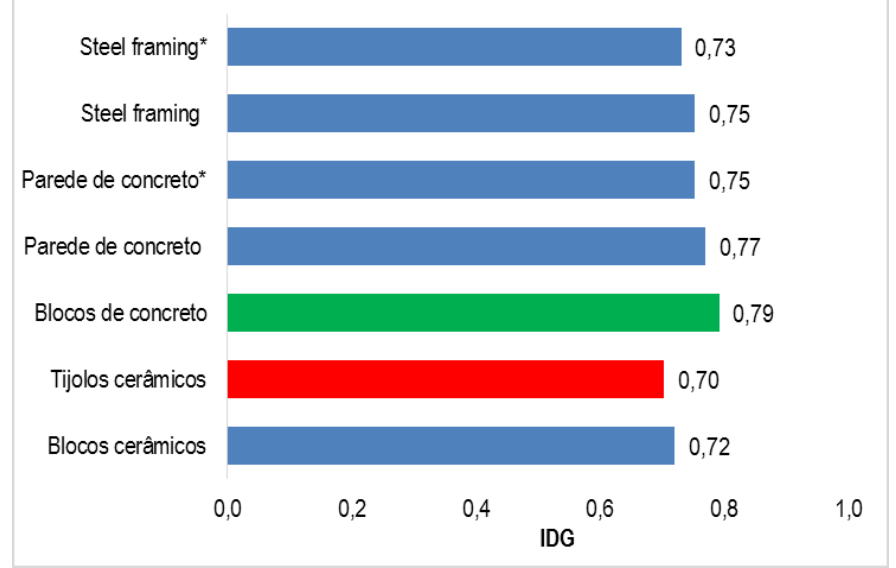

Fonte: Os autores.

Figura 6 - Comparação do indicador de desempenho global para o cenário 3 (com maior peso para os critérios térmicos)

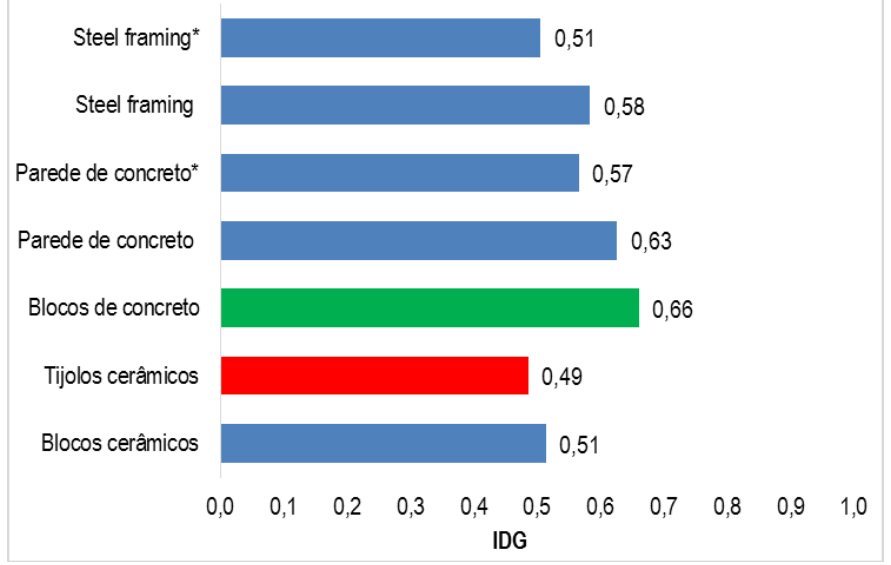

Fonte: Os autores.

Figura 7 - Comparação do indicador de desempenho global para o cenário 4 (com maior peso para os critérios térmicos (Ct>Rt))

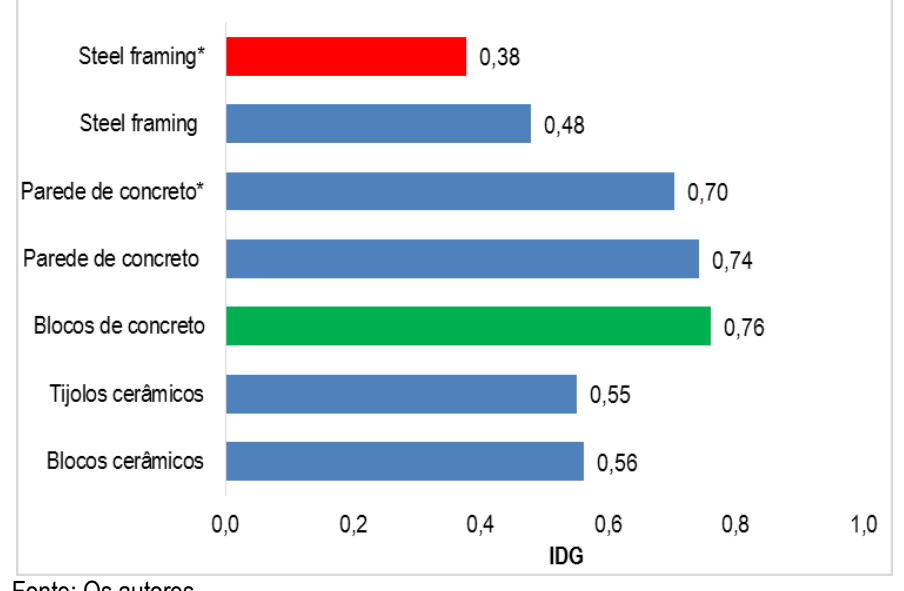

Fonte: Os autores.
Figura 8 - Comparação do indicador de desempenho global para o cenário 5 (com maior peso para os critérios térmicos $(\mathrm{Ct}<\mathrm{Rt}))$

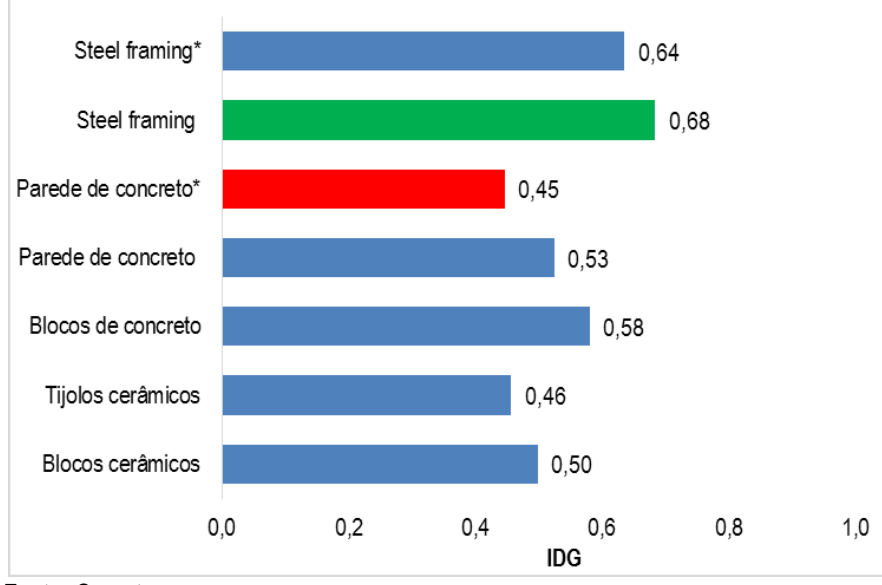

Fonte: Os autores.

Analisando os seis cenários é possível observar que o sistema de alvenaria de blocos de concreto apresenta os maiores IDGs, na maioria das vezes. Isso mostra que, com base nas premissas utilizadas neste estudo, este seria o sistema mais equilibrado em termos de desempenho acústico, térmico e ambiental, mesmo quando são dados diferentes pesos para os critérios.

Quando o desempenho acústico possui maior peso (Figura 5), o steel framing apresenta um aumento do seu IDG, ficando próximo do sistema de blocos de concreto, devido ao fato de possuir o maior $\mathrm{Rw}$.

No caso do desempenho térmico (Figura 6), o sistema de alvenaria de blocos de concreto também se destaca, já que possui valores de Rt e Ct intermediários. O steel framing possui uma elevada $\mathrm{Rt}$ mas uma $\mathrm{Ct}$ muito baixa, levandooa ter o pior IDG no cenário 4 (Figura 7).O oposto ocorre para a parede de concreto, que teve o pior IDG no cenário 5 (Figura 8).

Quando o desempenho ambiental é priorizado (Figura 9), o steel framing e a parede de concreto apresentam os menores valores de IDG, dado que são os que possuem piores indicadores de energia e emissões de carbono. Os sistemas de alvenaria de blocos e tijolos cerâmicos e de concreto apresentam valores de IDG aproximadamente iguais, com diferenças inferiores a $0,2 \%$.

Quando se avalia o IDG para os diferentes cenários verifica-se que a escolha do peso dos critérios, na matriz de decisão (Tabelas 3, 4, 5, 6,7 e 8), é um dos itens mais importantes.Tal escolha deverá ser fortemente influenciada pelas características do projeto em questão, como localização e orientação solar da edificação, características climáticas do local de implantação, necessidades específicas do cliente, entre outras.Por exemplo, para uma fachada de uma edificação que se encontra próxima a uma rodovia movimentada, é de se esperar que o critério acústico seja valorizado na escolha 
do sistema construtivo a ser utilizado, com um peso maior no AHP, que conforme exemplificado pelo cenário 1.

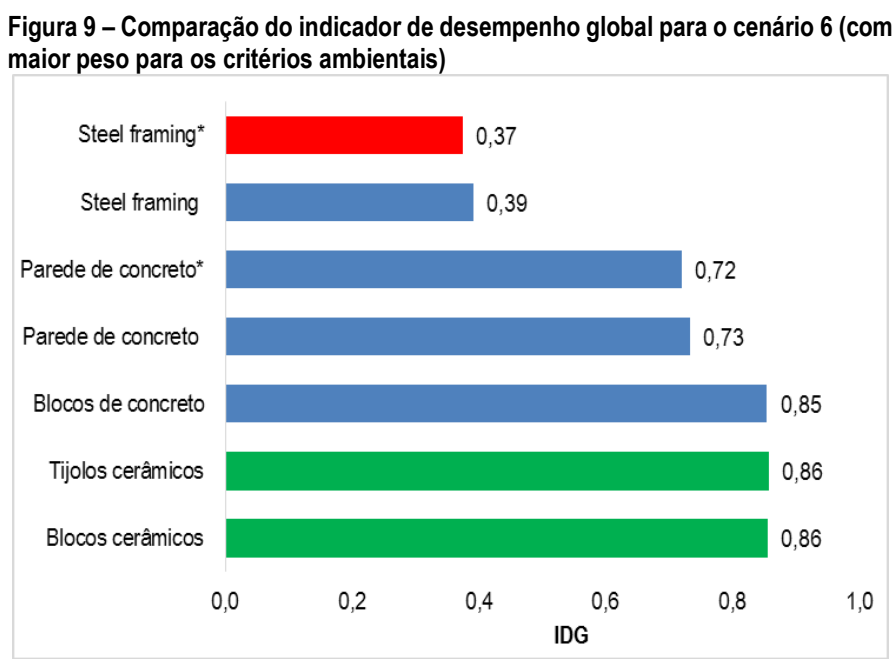

Fonte: Os autores.

Em um projeto localizado na Zona Bioclimática 4 (clima quente e seco), recomenda-se dar mais valor ao critério de capacidade térmica (exemplo do cenário 4), enquanto para uma localidade na Zona Bioclimática 8 (clima quente e úmido) deve-se priorizar o critério de transmitância térmica (exemplo do cenário 5), a fim de seguir as diretrizes da NBR 15220-3 (ABNT, 2005).

Um projeto com interesse por alguma certificação ambiental, como LEED ou AQUA, pode dar maior peso para indicadores ambientais, como o IE e o IC (exemplo do cenário 6).

A penalização dos sistemas construtivos que não atingiram critérios mínimos, como foi o caso da parede de concreto para resistência térmica e do steel framing para capacidade térmica, refletiu no valor do IDG, principalmente nos cenários 3, 4 e 5. Essa avaliação é importante, tendo em vista que,de acordo com o código brasileiro do consumidor (BRASIL, 1990), não se deve colocar no mercado produtos que não atendam aos requisitos $\mathrm{e}$ critérios mínimos das normas brasileiras.

Observa-se que o uso do método de AHP para a quantificação de um indicador global pode ser adaptado a

diferentes situações e projetos, além de permitir a incorporação de outros critérios no processo de avaliação, como custos, durabilidade e estanqueidade. Carvalho e Sposto (2012) propuseram uma metodologia similar, mas com foco específico para a sustentabilidade de habitações de interesse social, sem considerar critérios específicos da NBR 15575 (ABNT, 2013).

O presente estudo vai além do que foi realizado por Frazen (2015), que teve o foco específico para o sistema de steel framing, mostrando que é possível considerar outros aspectos de desempenho além do térmico e acústico, na avaliação de outros sistemas construtivos.

Observa-se assim o potencial de uso do método de AHPpara orientar e sistematizar escolhas de diferentes sistemas construtivos para vedações verticais.

\section{Conclusões}

Neste estudo foram avaliados o desempenho acústico, térmico e ambiental de diferentes sistemas construtivos comumente utilizados em fachadas de edificações no Brasil.

A partir de um indicador de desempenho global (IDG), que considerou critérios acústicos, térmicos e ambientais, verificou-se que a alvenaria de blocos de concreto é o sistema construtivo mais vantajoso para a maioria dos cenários avaliados.

O presente estudo traz três contribuições importantes: (1) a apresentação de resultados de indicadores ambientais para os principais sistemas construtivos de vedação vertical utilizados em edifícios habitacionais no Brasil, a partir da utilização de uma metodologia internacionalmente aceita, que pode ser utilizada em outros estudos, com as devidas adaptações, mesmo que exista a limitação do uso de banco de dados ambientais internacional de alguns materiais;(2) apresentação de uma método capaz de relacionar três diferentes critérios de desempenho para a avaliação de sistemas construtivos; (3) avaliação do potencial do uso do AHP para a tomada de decisão para a escolha de sistemas construtivos para vedações verticais.

Recomenda-se, para futuros estudos, que o cálculo dos critérios de desempenho acústico e térmico sejam realizados a partir de simulações computacionais, laboratório ou em obra. Seria interessante também incluir aspectos de custos, desempenho mecânico e durabilidade dos sistemas, o que deixaria a análise mais completa.

\section{Nota}

(1) Para o cálculo do $\mathrm{CO}_{2 \mathrm{e}}$ são considerados os valores de potencial de aquecimento global para 100 anos (GWP100) de 28 para $\mathrm{CH}_{4}$ e $265 \mathrm{~N}_{2} \mathrm{O}$ (IPCC, 2013). 


\section{Referências}

ABNT - ASSOCIAÇÃO BRASILEIRA DE NORMAS TÉCNICAS. NBR 15220-2: Desempenho Térmico de Edificações. Parte 2: Métodos de cálculo da transmitância térmica, da capacidade térmica, do atraso térmico e do fator solar de elementos e componentes de edificações. Rio de Janeiro, 2005.

ABNT - ASSOCIAÇÃO BRASILEIRA DE NORMAS TÉCNICAS. NBR 15575: Edificações Habitacionais - Desempenho. Rio de Janeiro, 2013

ABNT - ASSOCIAÇÃO BRASILEIRA DE NORMAS TÉCNICAS. NBR ISO 14040: Gestão ambiental - Avaliação do ciclo de vida - Princípios e estrutura. Rio de Janeiro: ABNT, 2009.

ANAND, Chirjiv K.; AMOR, Ben. Recent developments, future challenges and new research directions in LCA of buildings: A critical review. Renewable and Sustainable Energy Reviews, January, v. 67, n. 1, p. 408-416, jan. 2017. doi:https://doi.org/10.1016/j.rser.2016.09.058

ASTM - AMERICAN SOCIETY FOR TESTING AND MATERIALS. ASTM E 1765: standard practice for applying analytical hierarchy process (AHP) to multiatribute decision analysis of investments related to buildings and buildings systems. West Conshohocken, PA, 2002.

BRASIL. Código do Consumidor (1990). Lei no 8.078: promulgada em 11 de setembro de 1990. Disponível em: <http://www.planalto.gov.br/ccivil_03/leis/18078.htm> Acesso em 01 de abr. 2018.

CABEZA, Luiza, et al. Life cycle assessment (LCA) and life cycle energy analysis (LCEA) of buildings and the building sector: A review. Renewable and Sustainable Energy Reviews, v. 29, n.1, p. 394-416, jan. 2014 doi:https://doi.org/10.1016/j.rser.2013.08.037

CALDAS, Lucas R.; LIRA, Júlia S.; SPOSTO, Rosa M. Avaliação do Ciclo de Vida de habitações de alvenaria estrutural de blocos cerâmicos e painéis pré-moldados de concreto considerando diferentes zonas bioclimáticas. LALCA Revista Latino-Americana em Avaliação do Ciclo de Vida, v.1, n.1, p. 138 - 167, 2017a. doi:https://doi.org/10.18225/lalca.v1i1.3823

CALDAS, Lucas Rosse, et al. Life cycle carbon emissions inventory of brick masonry and light steel framing houses in Brasilia Proposal of design guidelines for low-carbon social housing. Ambiente Construído, v. 17, n. 3, jun. 2017 b. doi:http://dx.doi.org/10.1590/s1678-86212017000300163

CBIC - CAMARA BRASILEIRA DA INDÚSTRIA DA CONSTRUÇÃO. Desempenho de edificações habitacionais. Guia orientativo para atendimento a NBR 15575. Brasília, 2013.

CARVALHO, Michele T. M.; SPOSTO, Rosa M. Metodologia para avaliação da sustentabilidade de habitações de interesse social com foco no projeto. Ambiente Construído, v. 12, n. 1, p. 207-22, jan. 2012. doi:http://dx.doi.org/10.1590/S167886212011000200012

CHAU, C. K.; LEUNG, T. M.; NG, W. Y. A review on Life Cycle Assessment, Life Cycle Energy Assessment and Life Cycle Carbon Emissions Assessment on buildings. Applied Energy, v. 143, n. 1, p. 395-413, abr. 2015. doi:https://doi.org/10.1016/j.apenergy.2015.01.023

DARKO, Amos.; CHAN, Albert P. C.; AMEYAW, Ernest E.; OWUSU, Emmanuel K.; PÄRN, Erika.; EDWARDS, David J. Review of application of analytic hierarchy process (AHP) in construction. International Journal of Construction Management, v. 1, 2018. doi:https://doi.org/10.1080/15623599.2018.1452098

ECS - EUROPEAN COMMITTEE FOR STANDARDIZATION. CEN EN 15978: sustainability of construction works: assessment of environmental performance of buildings - calculation method. Brussels, 2011

FIGUEIREDO, Francisco, G., SILVA, Vanessa G. Processo de Projeto Integrado: recomendações para empreendimentos com metas rigorosas de desempenho ambiental. PARC Pesquisa em Arquitetura e Construção, Campinas, SP, v. 1, n. 5, p. 1-30, 2010. doi:https://doi.org/10.20396/parc.v1i5.8634493

FRANZEN, Fabiani P. Análise do desempenho térmico e acústico de vedações verticais externas executadas em light steel framing. 2015. 141 f.. Dissertação (Mestrado em Engenharia de Construção Civil) - Faculdade de Engenharia Civil, Universidade Federal do Paraná, Curitiba, 2015. 
GRÜNBERG, Paula R.; MEDEIROS, Marcelo H. F.; TAVARES, Sérgio F. Certificação Ambiental de Habitações: Comparação entre Leed for Homes, Processo Aqua e Selo Casa Azul. Ambiente \& Sociedade, São Paulo, SP, v. 17, n. 2, p. 195-214, 2014. doi:http://dx.doi.org/10.1590/S1414-753X2014000200013

IPCC - INTERGOVERNMENTAL PANEL ON CLIMATE CHANGE. Climate Change 2013: The Physical Science Basis. Contribution of Working Group I to the Fifth Assessment Report of the Intergovern mental Panel on Climate Change [Stocker, T.F., D. Qin, G.-K. Plattner, M. Tignor, S.K. Allen, J. Boschung, A. Nauels, Y. Xia, V. Bex and P.M. Midgley (eds.)]. Cambridge University Press, Cambridge, United Kingdom and New York, NY, USA, 1535 p. 2013.

INTERNATIONAL EPD SYSTEM. Disponível: <http://www.environdec.com/en/Detail/epd895> Acesso em 21. Jan. 2018.

IWARO, J.; MWASHA, A. The impact of sustainable building envelope design on building sustainability using Integrated Performance Model. International Journal of Sustainable Built Environment. v.2, p. 153-171, 2013. doi:https://doi.org/10.1016/j.ijsbe.2014.03.002

MARCHEZETTI, Ana A.; KAVISKI, Eloy.; BRAGA, Maria C. B.. Aplicação do método AHP para a hierarquização das alternativas de tratamento de resíduos sólidos domiciliares. Ambiente Construído, Porto Alegre, v. 11, n. 2, p. 173-187, abr./jun. 2011. doi: http://dx.doi.org/10.1590/S1678-86212011000200012

MATTANA, Alécio J.; MEDEIROS, Marcelo H. F. de; SILVA, Narciso G. da; COSTA, Marienne do R. de M. M. da. Análise hierárquica para escolha entre agregado natural e areia de britagem de rocha para confecção de argamassas de revestimento. Ambiente Construído, Porto Alegre, v. 12, n. 4, p. 63-79, out./dez. 2012. doi: http://dx.doi.org/10.1590/S167886212012000400006.

MMA - MINISTÉRIO DO MEIO AMBIENTE. Plataforma PROJETEE. Disponível em: <http://projeteee.mma.gov.br/componentes-construtivos/\#paredes> Acesso em 25 de jan. 2018.

PENEDO, Rafaella Cristina Teixeira ; OITICICA, Maria Lúcia G. Isolamento sonoro aéreo de partições verticais da sala de estar de um apartamento em Maceió-AL Brasil. PARC Pesquisa em Arquitetura e Construção, Campinas, v. 5, n. 2, p. 7-14, jul./dez. 2014. doi:https://doi.org/10.20396/parc.v5i2.8634533

RADAVELLI, G. F.; PAUL, S. Isolamento sonoro de parede em light steel frame com placas cimentícias vs. isolamento sonoro de paredes convencionais . In: ENCONTRO NACIONAL E IX ENCONTRO LATINO-AMERICANO DE CONFORTO NO AMBIENTE CONSTRUÍDO, 13., Campinas, 2015. Anais...Campinas: ANTAC, 2015. p. 1-10.

SAATY, Thomas L. The analytical hierarchy process. New York: McGraw-Hill, 1980.

SAATY, Thomas L. Some Mathematical Concepts of the Analytic Hierarchy Process. Behaviormetrika, v. 29, p. 1-9, 1991.

SACHT, Helenice M.; ROSSIGNOLO, João A. Habitações Térreas e Multipavimentos de Interesse Social: Avaliação de Desempenho Térmico para Tipologias com Vedações em Alvenaria de Blocos Cerâmicos e de Concreto. PARC Pesquisa em Arquitetura e Construção, Campinas, v. 1, n. 4, p. 7-14, jul./dez. 2009. doi:https://doi.org/10.20396/parc.v5i2.8634533

SAMPAIO, Juliana Carvalho Schlachter; LIMA, Mariana Monteiro Xavier de; BARROS NETO, José de Paula. A Utilização de Ferramentas de Apoio à Tomada de Decisão no Processo de Projeto. Gestão \& Tecnologia de Projetos, Brasil, v. 5, n. 1, p. 56-78, may 2010. doi:http://dx.doi.org/10.4237/gtp.v1i1.116

SANTOS FILHO, Vamberto M.; SPOSTO, Rosa M.; CALDAS, Lucas R. Análise do desempenho acústico de fachadas ventiladas de porcelanato à luz da norma de desempenho: estudo de caso em um edifício habitacional em Brasília-Df. REEC Revista Eletrônica de Engenharia Civil, Campinas, SP, v. 13, n. 1, p. 116-130, jul-dez 2017. doi:https://doi.org/10.5216/reec.v13i2.44959

SANTOS, Luciano F. S.; CRUZ, Rafael B. C. O Uso do Método AHP na Tomada de Decisão para Seleção de Sistemas de Lajes de Edifícios Comerciais. Engenharia Estudo e Pesquisa. ABPE, v. 13 - n. 1 - p. 39-52 - jan./jun. 2013.

SILVA JÚNIOR, Otávio Joaquim; RÊGO SILVA, José Jeférson; PINHEIRO, Marco Antonio Silva. Desempenho acústico de divisórias verticais em blocos de gesso: uma avaliação em campo e laboratório. PARC Pesquisa em Arquitetura e Construção, Campinas, v. 5, n. 2, p. 15-21, jul./dez. 2014. doi:https://doi.org/10.20396/parc.v5i2.8634534 
SINDUSCON - SINDICATO DA INDÚSTRIA DA CONSTRUÇÃO. Avaliação de Desempenho Acústico em diferentes sistemas construtivos, conforme a norma de desempenho ABNT NRB 15575/2013. Brasília, 2013.

SORGATO, M. J.; MELO, A. P.; MARINOSKI, D. L.; LAMBERTS, R. Análise do procedimento de simulação da NBR 15575 para a avaliação do desempenho térmico de edificações residenciais. Ambiente Construído, Porto Alegre, v. 14, n. 4, p. 83-101, out./dez. 2014. doi:http://dx.doi.org/10.1590/S1678-86212014000400007

SOUZA, Danielle Maia, et al. Comparative life cycle assessment of ceramic brick, concrete brick and cast-in-place reinforced concrete exterior walls. Journal of Cleaner Production, v.137, n. 20, p. 70-82, nov.2016.

doi:https://doi.org/10.1016/j.jclepro.2016.07.069

SOUZA, Henor A.; AMPARO, Lucas R.; GOMES, Adriano P. Influência da inércia térmica do solo e da ventilação natural no desempenho térmico: um estudo de caso de um projeto residencial em light steel framing. Ambiente Construído, v. 11, $\mathrm{n}^{\circ} 4$, out/dez. 2011. doi:http://dx.doi.org/10.1590/S1678-86212011000400009

TABORIANSKI, Vanessa. M.; PRADO, Racine. T. A. Methodology of $\mathrm{CO}_{2}$ emission in the life cycle of office building façades. Environmental Impact Assessment Review, v. 33. p. 41-47, 2012. doi:https://doi.org/10.1016/j.eiar.2011.10.004

TCPO - TABELA DE COMPOSIÇÃO DE PREÇOS PARA ORÇAMENTOS. TCPO 14. 14 ed. São Paulo: Pini, 2012.

\section{iLucas Rosse Caldas}

Engenheiro Civil, Ambiental e Sanitarista. Mestre em Estruturas e Construção Civil (PECC/UnB). Endereço Postal: Universidade Federal do Rio de Janeiro, Faculdade de Arquitetura e Urbanismo, Av. Pedro Calmon, 550, Cidade Universitária, Rio de Janeiro, RJ, Brasil, CEP: 21941-901

\section{ii Michele Tereza Marques Carvalho}

Engenheira Civil. Doutora em Estruturas e Construção Civil (PECC/UnB). Professora do Programa de Pós-graduação em Estruturas e Construção Civil (PECC), Departamento de Engenharia Civil e Ambiental da Universidade de Brasília. Endereço Postal: Campus Universitário Darcy Ribeiro, Asa Norte, Brasília, DF, Brasil, CEP: 70910-900 